\title{
Comparing the Quality of Life Before and After Total Hip Arthroplasty Operation in Patients With Developmental Dysplasia of the Hip
}

Fatemeh Sanei $^{1}$, Ali Asghar Jamebozorgi ${ }^{1 *}$, Ashkan Irani ${ }^{1}$, Alireza Akbarzade Baghban ${ }^{2}$, Mohammad Qoreishi ${ }^{3}$

1. Department of Occupational Therapy, Faculty of Rehabilitation, Shahid Beheshti University of Medical Sciences, Tehran, Iran. 2. Department of Basic Sciences, Faculty of Rehabilitation, Shahid Beheshti University of Medical Sciences, Tehran, Iran.

3. Department of Orthopedic Surgery, Akhtar Hospital, Shahid Beheshti University of Medical Sciences, Tehran, Iran.

Crtation: Sanei F, Jamebozorgi AA, Irani A, Akbarzade Baghban AR, Qoreishi M. Comparing the Quality of Life Before and After Total Hip Arthroplasty Operation in Patients With Developmental Dysplasia of the Hip. Physical Treatments. 2016; 5(4):219-224.

Article info:

Received: 18 May. 2015

Accepted: 20 Nov. 2015
Keywords:

Quality of life,

Developmental dysplasia

of the hip, Total hip

arthroplasty

\begin{abstract}
A B S T RA C T
Purpose: Developmental dysplasia of the hip (DDH) increases the risk of secondary degenerative changes and subsequent total hip arthroplasty. The postoperative quality of life in patients with $\mathrm{DDH}$ who have been born and grown with this disability and adapted to it during the life, is one of the most challenging issues. This study aimed to compare the quality of life of patients with DDH before and after total hip arthroplasty (THA) operation.

Methods: This research has a quasi-experimental/interventional design. A group of 20 patients with DDH, who underwent THA operation in Akhtar and Shafa yahyaiyan hospitals, were evaluated before operation and 6 months after it using SF36 quality of life questionnaire.

Results: Findings showed significant improvement in all SF-36 subscales scores, including physical function, role physical, role emotional, vitality, mental health, social function, bodily pain, general function, and general health as well as total score $(\mathrm{P}=0.002)$. SF-36 mean(SD) scores before and after surgery was 27.41(22.75) and 79.12(28.03), respectively.
\end{abstract}

Conclusion: THA operation improves the quality of life of patients with DDH.

\section{Introduction}

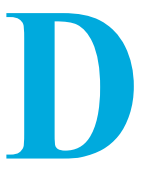

evelopmental dysplasia of the hip (DDH) refers to a wide spectrum of conditions ranging from dysplasia to mild and complete dislocations of hip joint [1]. This disorder is one of the most common congenital diseases associated with socioeconomic consequences [2]. If non-surgical treatment begins before the 6 months age the outcome will be successful. On the other hand, delayed diagnosis may result in the complex surgery management [3].

\section{* Corresponding Author:}


Total hip arthroplasty (THA) is one of the treatment options for DDH [4]. In recent years, THA operation has drawn the attention of many scholars and physicians, and the demand for this operation is increasing [5]. Today, THA operation is widely undertaken throughout the world. It is estimated that approximately 170000 people in the United States and 300000 people around the world are undergoing this operation every year [6]. This type of operation has an extraordinary evolution from a procedure with poor level of long-term outcomes applied for severe disabled patients to a most effective and most commonly used surgical procedures performing to relieve pain and restore the function of hip joint [7].

Reconstruction of hip joint after surgery is difficult because of significant differences in hip anatomical abnormalities associated with different types of DDH (dysplasia, mild dislocation, and severe dislocation) [8]. Though primary THR can significantly improve function in patients, studies have reported high rate of postoperative complications such as implant loosening and failure, infection, pain and anxiety, fear of falling and weakness in the muscles of the hip. These complications affect the quality of life, functional activities, and independence of the patients [9].

There are a number of challenges following THA operation that changes in the "quality of life" can be one of them. It is difficult to define the "quality of life," however for most people, it is a broad concept encompassing satisfaction in all aspects of life such as health, employment, housing, and education. Although the life expectancy of patients has been previously important, now a lot of attention is being paid on the "quality of life". Health-related "quality of life" is a condition in which the patient is satisfied with emotional, social, and physical functioning aspects of life. Whatever is eventually achieved and sustained for the patient following a medical or surgical intervention would be the best and most essential criterion for judging the effectiveness of that intervention [10].

The factors affecting the "quality of life" include individual, social, psychological, and demographic factors, such as gender, marital status, education, and physical health status. In addition, in the elderly the development of different types of fractures and deformities and arthritis can be noted. In people over age 60, fall-related fractures are the most common cause of mortality which can negatively impact their "quality of life" [11, 12, 13, 14].

Questionnaires should measure the items that the patient be able to answer with regard to treatment outcomes. Several questionnaires have been designed for this purpose, including the 36-item Short Form Health Survey
(SF-36) that is a generic tool to measure health status and "quality of life" in both healthy and sick people [15].

Quality of life of the patients with DDH treated with THA is different from those suffering from other hip diseases. Because such patients have been born and grown with this disability, and in a society where the physical ability is a privilege, their lives have been affected substantially.

These patients, besides going through long and multiple treatment experiences, have traumatic experiences, too. For example over the time, with progressing the symptoms, changes in their appearances occur, so that the affected leg rotates externally and looks short, and distortion of posture and asymmetry in the back, shoulder, waist, and hips occur. Sometimes the muscles atrophy is obvious, too. Moreover, such patients assume themselves disabled because of their difficulty in movement and deformed body. Negative attitudes of people toward disability and their abnormal gait and posture lead to their anxiety and social separation and consequently affects their development of body image and positive self-concept.

All these problems affect socially, mentally, and emotionally the patient's life and so the surgical treatment is chosen to get rid of these problems. On the other hand, the operation is an invasive method. This method includes leaving scars around the hips and thighs, in-vivo hip screw, metal sheets and metal joint that especially creates mental problems for the young women who are concerned about their appearances. Furthermore, operation includes a long rehabilitation program causing anxiety in the patient. Although, operation alone can be successful; physical, clinical, and high mental adaption are essential for postoperative recovery, especially in the women and those with severe disability who may be more vulnerable and prone to psychosocial problems and suffer from postoperative long-term problems [15].

In addition, significant difference in hip anatomical abnormalities associated with different types of DDH (dysplasia, low dislocation, high dislocation) causes difficulty in reconstruction using TTA operation [8]. Abnormal bone structures, failure in positioning of the hip, limb length differences, abnormal neurovascular structures, dysfunctional abductor muscles, especially poor acetabular bone stock and the inner cannula narrow femoral medullary create technical problems [4]. As a result, all these problems create postoperative problems for the "quality of life" in patients with DDH to a challenging degree that requires more research.

Although there are many studies about the "quality of life" in patients with different types of diseases related 
to hip joint, very limited research has been conducted in this special group of patients with DDH after total joint replacement. Little evidence in this area results in low awareness of medical and rehabilitation team about expectations and emotions of these patients. Therefore, the necessary post- and pre-operative psychosocial interventions are not taken into account and accurate selection of surgical candidates is not performed. Also, lack of awareness of the complications and possible problems related to the operation, may lead to the irreparable consequences. Despite the importance of this subject and lack of knowledge about all aspect of its complications, so far no study in Iran has been done in this area and there is only a limited number of the foreign research about it.

Therefore in this study, we compared pre- and post-operative "quality of life" in patients who underwent THA operation with anterolateral approach and the effect of this operation on the "quality of life" to obtain accurate information about postoperative expectations and emotions of these patients, and based on this information, we can take action and make relevant medical, rehabilitation, and psychological decisions and design necessary trainings.

\section{Materials and Methods}

This study had a quasi-experimental/interventional design and 20 patients aged 30-70 years participated in it. They were selected based on the inclusion criteria, which included undergoing the primary THA operation and lacking history of any previous operation. Written consent forms were obtained from all the participants and preoperative assessment using the SF36 questionnaire was done by occupational therapists of the research team in department of occupational therapy of Akhtar and Shafa yahyaian hospitals, Tehran, Iran.

Because of shortness and comprehensiveness of the SF-36 questionnaire, it is one of the most frequently used instruments to assess the health status and "quality of life" in the world. It has been used in over 4000 published studies. SF-36 questionnaire includes some statements determining subscales of physical functioning, role physical, bodily pain, general health, vitality, social functioning, role emotional, mental health, and one statement relating to health status changing. After the initial assessment, patients were undergone surgery by orthopedic surgeon in Akhtar or Shafa yahyaian hospital with anterolateral approach. After operation, all patients received rehabilitation program in the hospital, in accordance with the protocol of rehabilitation following THA operation in the Orthopedic Rehabilitation text- book which was approved by all occupational therapists of the team and was consistent with the their approach.

Patients were contacted 6 months after operation and the SF-36 questionnaire was re-completed by a telephone interview and based on the instructions scoring. The preoperative and postoperative results were compared. Standard deviation and variations were calculated for each sample and the normality distribution of the data and the samples using the one sample Kolmogorov-Smirnov was determined. Wilcoxon signed rank test was used to compare the scores. Level of significance was considered 0.05 and data analysis was performed using the SPSS 18 .

\section{Results}

Of 20 cases, one patient was unavailable to complete the questionnaire after operation and one patient was excluded because of damage to the operated joint. Therefore, the study group comprised 18 patients included 16 females and 2 males, average age 43.89 years (range: 31 to 56 years). Follow-up time was 6 months. Table 1 shows the results of SF-36.

Using non-parametric Wilcoxon test, the "quality of life" of the patients before and after operation was compared. Significant differences were observed in all domains of SF-36. Differences between means showed significant improvements in all aspects of "quality of life".

\section{Discussion}

Nowadays, generic utility measures of the "quality of life" beyond the traditional assessment tools increase in the literature, because through these indicators the surgical decision-making is not only based on the physical and radiologic findings but also based on the "quality of life" of patients. The "quality of life" assessments should not replace the clinical and radiographic examinations and should not be used alone for recognizing the surgical indication. The "quality of life" measuring can help identify the patients' preoperative expectations and emotions about joint replacement [16]. What finally can be achieved and remain for patient after a medical treatment or an operation is the best and the most essential criterion for judging the effectiveness of a treatment [9].

Several studies have reported the effect of THA operation on the "quality of life" of patients. In a study by C.Y. Ng et al. (2007), postoperative "quality of life" and functional outcomes after surgery were evaluated. The results showed significant improvements in all domains of SF-36 except for mental health and general health. 
Table 1. The results of SF-36.

\begin{tabular}{ccccccc}
\hline & Preoperative Score Mean & SD* & Postoperative Score Mean & SD & Mean Differences & P-value \\
\hline Physical functioning & 33.88 & 30.84 & 71.94 & 29.90 & 38.05 & 0.009 \\
\hline Role physical & 6.94 & 23.95 & 75.00 & 40.21 & 68.05 & 0.000 \\
\hline Role emotional & 29.62 & 45.57 & 88.88 & 32.33 & 59.25 & 0.001 \\
\hline Vitality & 31.38 & 33.46 & 77.50 & 34.00 & 46.11 & 0.004 \\
\hline Mental health & 44.44 & 32.26 & 83.70 & 29.89 & 35.77 & 0.007 \\
\hline Social functioning & 43.75 & 35.42 & 88.19 & 32.21 & 44.44 & 0.001 \\
\hline Bodily pain & 6.80 & 14.59 & 79.30 & 27.38 & 79.50 & 0.000 \\
\hline General health & 22.50 & 11.14 & 71.94 & 25.21 & 49.44 & 0.000 \\
\hline Total score & 27.41 & 22.75 & 79.12 & 28.03 & 51.70 & 0.000 \\
\hline *SD: Standard Deviation & & & & PHYSICAL TREA MENTS
\end{tabular}

*SD: Standard Deviation.

PHYSICAL TREA T MENTS

Also the improved outcomes were sustained during the follow up [17]. In another study, Susanne Knutsson et al. investigated the postoperative changes in the "quality of life" of patient and demonstrated the significant differences in patient's general, physical, and psychosocial "quality of life" after THA operation compared with the preoperative condition [18].

Although there are several studies about the "quality of life" of patients after THA operation, this study is one of the few studies that evaluated "quality of life" in a specific group of patients. In Iranian studies, there is no study conducted on evaluating the "quality of life" in patients suffering from DDH after THA operation comparing with the situation before the operation, and in the foreign studies there are only a limited number of studies that will be discussed in the following paragraphs.

Nikolaos T. Roidis studied the "quality of life" after THA operation and came into conclusion that THA in congenital hip dislocation (CHD) result in entirely longterm improvement in the "quality of life" [19]. Alessandra Tellini et al. (2008) in the article entitled" Quality of life evaluation in patients affected by osteoarthritis secondary to congenital hip dysplasia after total hip replacement, reported the improvement in the "quality of life". In another exploring study, it was noted that treatment of DDH requires much effort, resilience, and commitment [20].

In the current study, an obvious increase in SF-36 mean scores from 27.41 to 79.12 indicated that the "quality of life" of patients with DDH after THA operation significantly improved. This improvement was observed in all aspect of quality of life, including physical functioning, role physical, role emotional, vitality, mental health, social functioning, bodily pain, and general health, as well as total score. The most improvement was observed in the bodily pain, so that the mean difference after and before the operation was 79.50. Since the anatomical abnormalities lead to secondary degenerative changes of joint and these changes cause pain [21], it seems that the changes occurring in anatomical structures of joint following operation result in reduction of the pain and improvement in this domain that is higher and more objective than other domains.

Role physical subscale is the second domain with the most improvement that can be related to tendency of patient to exposure their recent physical condition better than the time before the operation [16]. Also, one of the reasons which causes higher patient's scores in these two subscales, is improvement in gait. The improved gait increases the concept of their role and their real physical function in daily life [21].

Jones et al. administered the SF-36 questionnaire in 208 patients with DDH 1 month and 6 months after THA operation. Much improvement in physical function, role physical, and bodily pain was observed that is consistent with the current study [21]. In Tellini study, after physical function, the highest improvement was observed in two mentioned domains [16].

The difference of present study with other studies was that in other studies the highest improvement was observed in physical function but such a result was not obtained in the current study that may be related to importance of the culture in this study and development 
of assistive equipment in other studies. For example, in our country and with regard to the culture, sitting on the ground or using the Iranian toilet is very important for individuals, but in the developed countries after the hip replacements operation, the individuals have less problem because of the assistive equipment such as toilet seat lift, traffic path without obstructing at home and outside the home, wedge between the legs of the chair and the likes. Another reason can be the precautions after the operation. Many participants of the this study when filling out the SF-36 questionnaire mentioned that precautions after operation restricted or changed some of their activities (limitation of the flexion of the hip to 90 degrees, adduction of the hip to 0 degrees, internal rotation to 0 degree). For example, they had difficulty in doing heavy activities such as running and lifting heavy objects because of the precautions after operation. Furthermore, the low score of these subscale scores may indicate that the patients after operation think that they are limited in some activities of daily living [21].

Consistent with the results of other studies, the minimum difference in mean scores before and after operation was observed in mental health domain (mean differences: 35.77 ). The reason for this is that preoperative mean score in this domain was the highest among all preoperative mean scores. According to the present study, $77 \%$ of the 18 cases were above 40 years old and it can be concluded that older patients more than younger counterparts could be adapted to the functional limitations [16] which result in less impact on mental health and getting higher preoperative score in this domain and consequently the mean difference before and after the operation reduced.

Comparing the questionnaire components summary results, namely the physical and mental components, revealed that improvement in physical function domain was higher than mental domain. So, the mean difference before and after operation with regard to the physical components was 57.1 and with regard to mental component 92.49.

The higher score in the physical components can be related to the lower comorbidities among the people aged less than 65 years, (according to the mean age of this study population). As a result, the individuals get higher score in physical domains which general health is one of its subscales [16].

Lower score in mental component may be related to this issue that the women under 65 years suffer more from chronic diseases, compared to the men, and have more mental disorders [21]. Therefore the overall results can be generalized to this gender, as most study population are women. Also, the reason for this can be the higher patient's expectation from the results of the operation and their viewpoint on the effect left by the operation and the insufficient clarification and explanations by the surgeons about the final result of the operation.

One of the limitations of our study was that most patients were female, therefore we could not compare the two genders. Furthermore, the study population was selected from Tehran, therefore the results cannot be generalized to the whole Iran society.

Our results indicated that THA operation in patients with DDH significantly improves the "quality of life" among them. This improvement has been observed in all aspects of "quality of life," including physical function, role physical, role emotional, vitality, mental health, social function, bodily pain, general function, and general health.

\section{Acknowledgements}

The current research hasn't received any financial support.

\section{Conflict of Interest}

The authors declared no conflict of interests.

\section{References}

[1] Boyle MJ, Frampton CM, Crawford HA. Early results of total hip arthroplasty in patients with developmental dysplasia of the hip compared with patients with osteoarthritis. Journal of Arthroplasty. 2012; 27(3):386-90. doi: 10.1016/j. arth.2011.06.024

[2] Clarke N. Swaddling and hip dysplasia: an orthopaedic perspective. Archives of Disease in Childhood. 2014; 99(1):5-6 doi: 10.1136/archdischild-2013-304143

[3] Fitch RD. Ultrasound for screening and management of developmental dysplasia of the hip. North Carolina Medical Journal. 2014; 75(2):142-45. doi: 10.18043/ncm.75.2.142

[4] Erdemli B, Yilmaz C, Atalar H, Güzel B, Cetin I. Total hip arthroplasty in developmental high dislocation of the hip. Journal of Arthroplasty. 2005; 20(8):1021-028. doi: 10.1016/j. arth.2005.02.003

[5] Vahid Farahmandi M, Esmailijah A, Madadi F, Abassian M, Zanganeh R, Valaie N, et al. [Results of total hip arthroplasty and its associated factors in Akhtar hospital (Persian)]. Research in Medicine. 2010; 33(4):241-46.

[6] Li H, Wang L, Dai K, Zhu Z. Autogenous impaction grafting in total hip arthroplasty with developmental dysplasia of the hip. Journal of Arthroplasty. 2013; 28(4):637-43. doi: 10.1016/j.arth.2012.07.007 
[7] Pivec R, Johnson AJ, Mears SC, Mont MA. Hip arthroplasty. Lancet. 2012; 380(9855):1768-777. doi: 10.1016/s01406736(12)60607-2

[8] Kim YH, Kim JS. Total hip arthroplasty in adult patients who had developmental dysplasia of the hip. Journal of Arthroplasty. 2005; 20(8):1029-036. doi: 10.1016/j.arth.2004.06.033

[9] Bozorgi J, Asghar A, Ghamkhar L, Kahlaee AH, Sabouri H. The Effectiveness of Occupational Therapy Supervised Usage of Adaptive Devices on Functional Outcomes and Independence after Total Hip Replacement in Iranian Elderly: A Randomized Controlled Trial. Occupational Therapy International. 2015; 23(2):143-53. doi: 10.1002/oti.1419

[10] Naseri MH, Panahi F, Motififard M, Teimouri M. [Quality of Life and Functional Outcome after Total Hip Arthroplasty (Persian)]. Journal of Isfahan Medical School. 2010; 27(102):733-41.

[11] Asadi M, Shiralipur A, Ghanad F, Nazary A. [Meta-analysis of effective factors in life quality of special patients (Persian)]. Journal of Hormozgan University of Medical Sciences. 2012; 16(1):25-33.

[12] Arastoo A, Ghassemzadeh R, Nasseh H, Kamali M, Rahimi FA, Arzaghi M, et al. [Factors affecting quality of life in elderly diabetic residents of the Kahrizak Geriatric Nursing Home of Tehran (Persian)]. Iranian Journal of Endocrinology And Metabolism. 2012; 14(1):18-24.

[13] Choobineh A, Daneshmandi H, Parand M, Ghobadi R, Haghayegh A, Zare F. [The Survey of Quality of Work Life and Determination of Its Related Factors in Shiraz University of Medical Sciences Staff (Persian)]. Journal of Ergonomics. 2013; 1(2):56-62.

[14] Jamebozorgi AA, Kavoosi A, Shafiee Z, Kahlaee AH, Raei $M$. Investigation of the prevalent fall-related risk factors of fractures in elderly referred to Tehran hospitals. Medical Journal of The Islamic Republic of Iran. 2013; 27(1):23-30. PMID: 23483674

[15] Gambling TS, Long AF. Experiences of young women living with developmental dysplasia of the hip: insight into their experiences of surgery and recovery. Chronic Illness. 2013; 9(1):16-28. doi: 10.1177/1742395312450177

[16] Motamed N, Ayatollahi A, Zare N, Sadeghi Hassanabadi A. Validity and reliability of the Persian translation of the SF-36 version 2 questionnaire. Eastern Mediterranean Health Journal. 2005; 11(3):349-57. PMID: 16602453

[17] Tellini A, Ciccone V, Blonna D, Rossi R, Marmotti A, Castoldi F. Quality of life evaluation in patients affected by osteoarthritis secondary to congenital hip dysplasia after total hip replacement. Journal of Orthopaedics and Traumatology. 2008; 9(3):155-58. doi: 10.1007/s10195-008-0022-6

[18] Ng C, Ballantyne J, Brenkel I. Quality of life and functional outcome after primary total hip replacement: a five-year follow-up. Journal of Bone \& Joint Surgery. 2007; 89(7):86873. doi: 10.1302/0301-620x.89b7.18482

[19] Knutsson S, Engberg IB. An evaluation of patients' quality of life before, 6 weeks and 6 months after total hip replacement surgery. Journal of Advanced Nursing. 1999; 30(6):1349-359. doi: 10.1046/j.1365-2648.1999.01232.x

[20] Roidis NT, Pollalis AP, Hartofilakidis GC. Total hip arthroplasty in young females with congenital dislocation of the hip, radically improves their long-term quality of life. Journal of Arthroplasty. 2013; 28(7):1206-211. doi: 10.1016/j. arth.2012.09.025

[21] Sliwinski MM, Sisto SA. Gait, quality of life, and their association following total hip arthroplasty. Journal of Geriatric Physical Therapy. 2006; 29(1):8-15. doi: 10.1519/00139143200604000-00003 